\title{
OBSERVATIONS OF ENERGETIC X-RAYS \\ FROM QUIESCENT SOLAR ACTIVE REGIONS*
}

\author{
LOREN W. ACton and Philip C. Fisher ** \\ (Lockheed Palo Alto Research Laboratory, Palo Alto, Calif., U.S.A.)
}

\begin{abstract}
A BST RACT
A measurement of the solar X-ray spectrum in the $1-30 \mathrm{KeV}$ interval was made by rocket-borne instruments at 0015 UT on April 5, 1967. These measurements show that the solar X-ray spectrum, which falls off steeply with energy below $4 \mathrm{KeV}$, becomes less steep above this energy. These data are taken to indicate that energetic, non-thermal, electrons are present or continuously produced in quiescent solar active regions. No optical flare activity was reported for several hours around the time of these observations.
\end{abstract}

\section{The Experiment}

A solar active region represents a remarkable concentration of energetic phenomena exemplified by the tremendous outpouring of energy associated with solar flares which, although long recognized, still remain to be understood. A study of the quiescent state of active regions can advance our knowledge of the physical nature of such regions and hence the sources of flare energy.

An experiment to investigate the quiescent state of solar active regions was carried out by instruments flown on a spin-stabilized rocket, which was launched from White Sands Missile Range, N.M., at 0015 UT on April 5, 1967. The nose of the spinning rocket was pointed approximately at the zenith with a yaw cone having a half angle of about $5^{\circ}$. X-ray detectors were mounted so as to look out the sides of the rocket. The Sun was located in the West at a zenith angle of approximately $75^{\circ}$. The experiment did not provide any information concerning the positions of the X-ray sources on the Sun. However, recent X-ray photographs of the Sun (Giacconi et al., 1965; Pounds and Russell, 1966; Underwood and Muney, 1967; Zhitnik et al., 1967) seem to establish conclusively that the emission of the harder X-rays is restricted to centres of activity.

The object of the experiment was to measure the solar X-ray emission in the $1 \mathrm{KeV}$ $(12 \AA)$ to $30 \mathrm{KeV}(0.4 \AA)$ region. The radiation sensors were eight gas-filled proportional counters with a total area of $0.34 \mathrm{~m}^{2}$. The signals from these proportional

* This work has been supported by the National Aeronautics and Space Administration under contract NASw-1398 and by the Lockheed Independent Research Program.

** Presented by L. W. Acton.

Kiepenheuer (ed.), Structure and Development of Solar Active Regions, 432-438. C. I.A.U. 
counters were processed by five different pulse-amplitude measuring systems which were equipped with a variety of provisions for reducing the deleterious effects of the cosmic-ray environment. The primary instrument, which yielded the most precise measurement of the solar X-ray spectrum, consisted of four proportional counters of total area $0.23 \mathrm{~m}^{2}$. Figure 1 illustrates the computed photon-detection efficiency

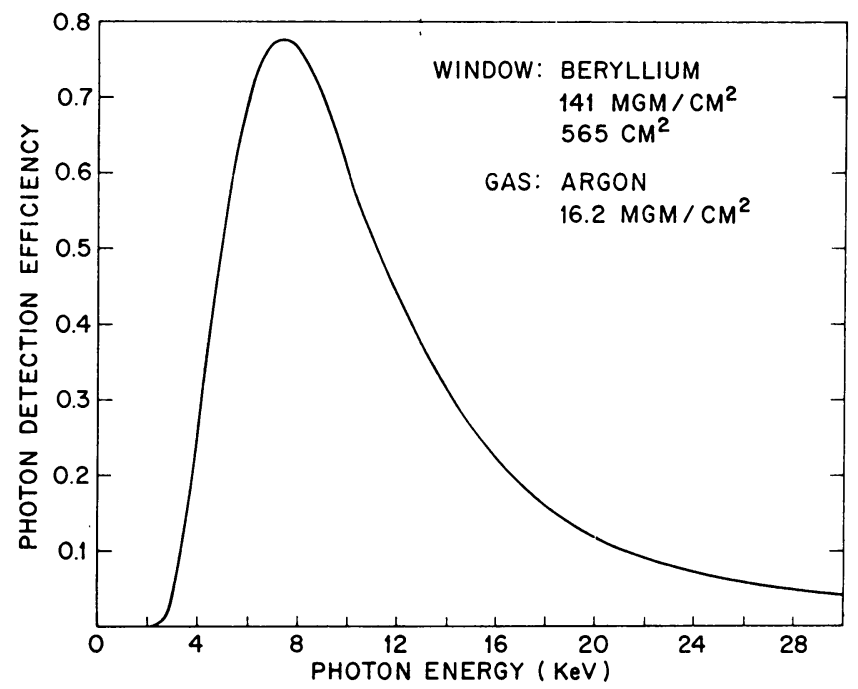

FIG. 1. Typical photon-detection efficiency of the proportional counters used in the primary system. Four such counters were used to feed the primary pulse-height analyzer. The energy resolution (full width at half maximum) of these counters at $6 \mathrm{KeV}$ was better than $20 \%$ over the entire window area.

of each of the four counters of the primary instrument. These counters were equipped with field-shaping electrodes so that the gain and resolution were uniform to within a few percent over the entire window area. Pulses from these counters were fed into a single analog-to-digital pulse-height analyzer which was developed specifically for this experiment (Reed et al., 1968). The analyzer was designed so that high pulse rates could be handled and a complete 16-channel spectrum was read out every 5 milliseconds, with no dead time introduced by the read-out process. The channels of this analyzer were arranged so that the channel widths in the $3-8.5 \mathrm{KeV}$ interval were approximately $0.5 \mathrm{KeV}$. The higher energy channels were set wider because of the anticipated lower photon flux at these energies. A very important feature of this X-ray detection system was a pulse rise-time discrimination circuit which rejected a high percentage of the pulses produced in the proportional counters by cosmic rays while accepting the pulses produced by X-rays. This circuit was developed especially for this experiment by Dr.R.C. Catura of this laboratory. The technique was first used by Mathieson and Sanford (1963). 


\section{The Data}

It is important to emphasize that the following discussion of the experimental results represents a preliminary analysis of the data from the flight. The possibility exists that systematic errors remain, both in our understanding of the performance of the instruments and in the data-reduction techniques which have been used. However, the internal consistency of the initial reduction of these data is such that preliminary conclusions may be drawn at this time.

Figure 2 presents examples of the azimuthal distribution of detector counts recorded in three different pulse-height intervals, or channels, of the primary instrument. These data were acquired in $94 \mathrm{sec}$ and represent a sum over 176 rolls of the spinning rocket.

Most of the non-solar or 'background' signal is caused by diffuse cosmic X-rays and unresolved cosmic $\mathrm{X}$-ray sources. The sinusoidal variation with azimuth of this signal is introduced by the tilt, toward the North, of the rocket roll axis. Channels 5 and 15 have a distinct maximum in the West, the position of the Sun at the time of the flight. The data in the figure have not been corrected for system dead-time.

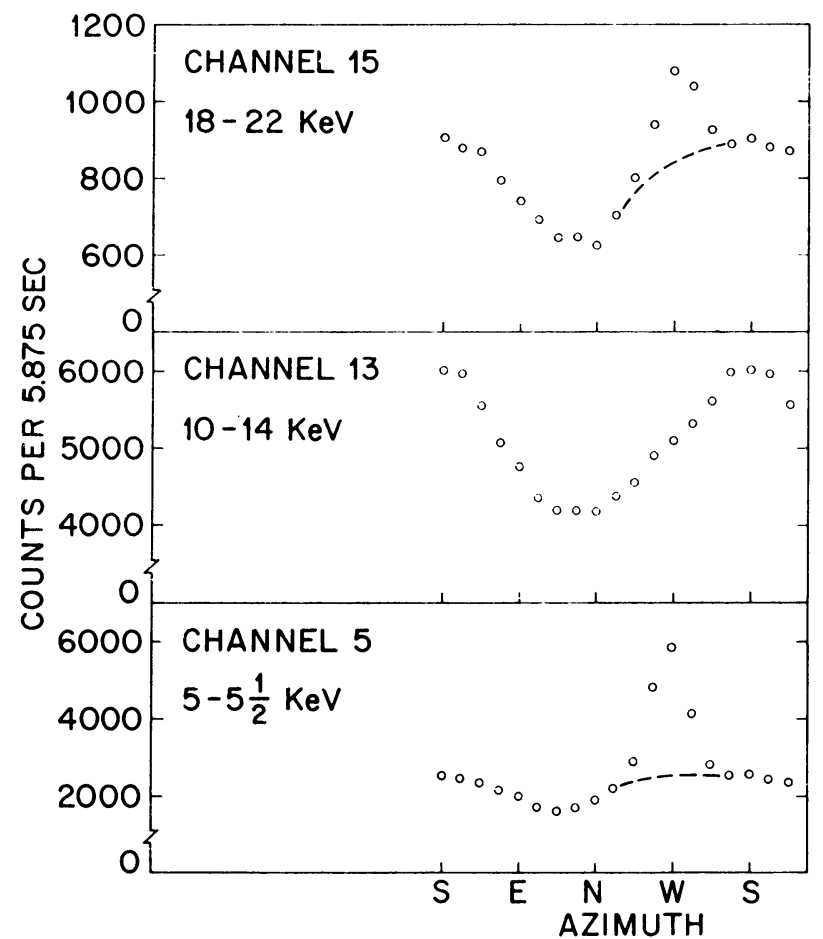

FIG. 2. Azimuthal distribution of signals from three channels of the primary system summed over 176 rolls of the spinning rocket. The width of the solar peak corresponds to the detector field of view in the direction normal to the spin axis of the rocket. 
The solar signal is not evident in channel 13 where the signal to background ratio is poorer than in the other channels.

A preliminary solar spectrum, derived from a partial reduction of the flight data, is presented in Figure 3. The statistical uncertainties of these data are small, and in most cases are roughly the size of the points which have been plotted. However, with the exception of the measurements below $3 \mathrm{KeV}$, the data have not been corrected

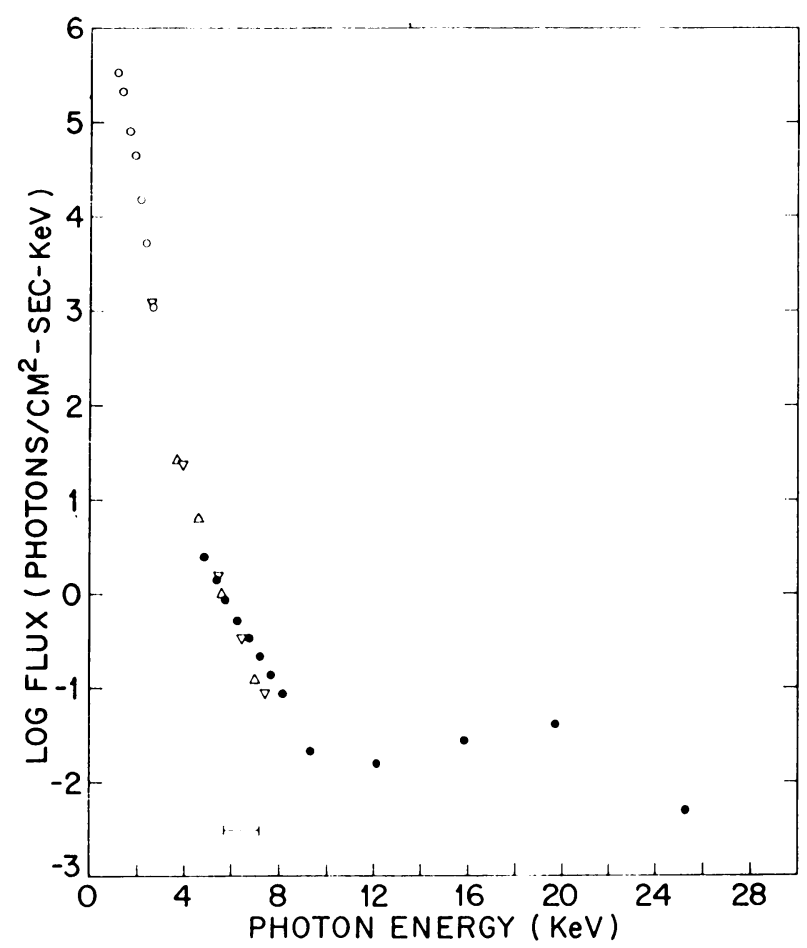

FIG. 3. Solar X-ray spectrum derived from a preliminary analysis of a portion of the data from four of the five X-ray detection systems flown on April 5, 1967. The data from each system are indicated by different symbols. Those points from the primary system are represented by filled circles. With the exception of the data indicated by the open circles, none of the data have been corrected for system dead-time. It is anticipated that the properly corrected data will not exhibit a pronounced minimum in the spectrum in the 8-14 KeV interval. The horizontal bar indicates the energy resolution (full width at half maximum) of the primary system at $6.4 \mathrm{KeV}$.

for the dead-time of the pulse-amplitude measuring systems. These corrections are in general less than $20 \%$, but they may significantly alter the appearance of the spectrum at the higher energies. We anticipate that the apparent minimum in the spectrum in the $8-14 \mathrm{KeV}$ interval will disappear or become less pronounced when the corrections for dead-time are properly taken into account. 


\section{The Interpretation}

Figure 4 is a reproduction of a Sacramento Peak Observatory $\mathrm{H} \alpha$ filtergram taken approximately $9 \frac{1}{2}$ hours prior to the time of the flight. At the time of the flight there was a complex of old active regions on the Sun in the Northern hemisphere. There were also four active regions in the Southern hemisphere, two of which produced the only flares within 12 hours of our flight. The two extremely active regions that had crossed the disk during the two weeks prior to the flight had passed the Western limb two days earlier. It is possible that some of the X-ray emission recorded by the rocket instruments came from the corona overlying these two regions.

It seems unlikely that 'flare' X-rays contributed to the spectrum of Figure 3 . There were no reported flares or sub-flares within the time interval of 3 hours prior to flight time to 7 hours after the time of the flight.

While it is inappropriate to draw extensive conclusions from these preliminary results the following features may be mentioned. The measurements of the solar

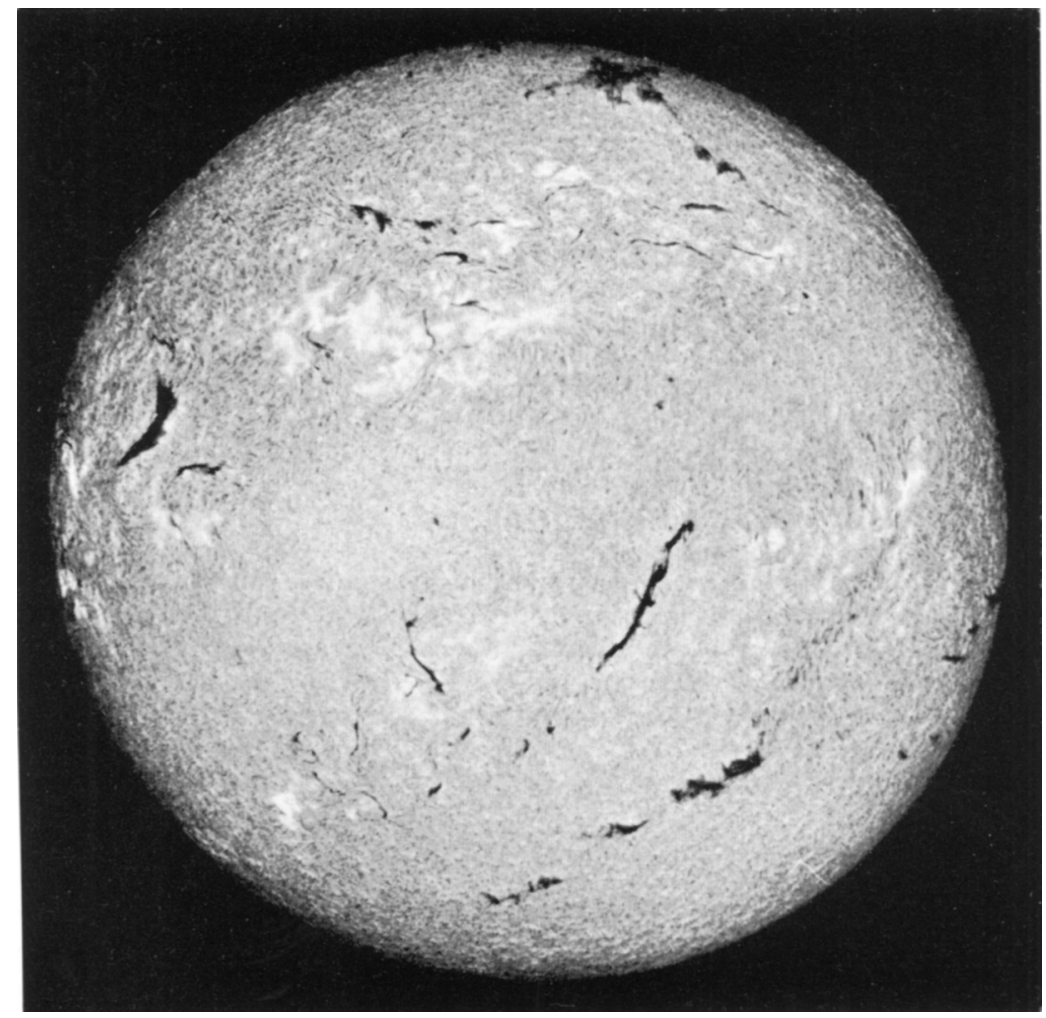

Fig. 4. Ho filtergram of the Sun taken at 1448 UT on April 4, 1967. (Courtesy of Sacramento Peak Observatory.) 
spectrum at energies below $4 \mathrm{KeV}$ are consistent with those of other workers (Bowles et al., 1967). The observed X-ray intensity lies between that reported by Chodil et al. (1965) and that of Underwood given by Bowles et al. (1967). Fritz et al. (1967) have shown that the $\mathrm{X}$-ray spectrum at energies below $6 \mathrm{KeV}$ is dominated by emission lines.

The slope of the $\mathrm{X}$-ray spectrum above $4 \mathrm{KeV}$ in energy is markedly less steep than at the lower energies. A second change in the nature of the solar X-ray spectrum appears to occur in the $8-12 \mathrm{KeV}$ interval where the spectrum becomes approximately flat, extending to the upper limit of the most reliable data at $22 \mathrm{KeV}$. The flux which we observed around $15 \mathrm{KeV}$ is almost two decades higher than the upper limit given by Peterson et al. (1966) for February 18, 1966 and about five decades below that reported by Chubb et al. (1966) from their pioneering observations made in 1959 . However, our data appear to be consistent with the measurements reported by Peterson (1967) for the 7.7-12.5 KeV and $12-22 \mathrm{KeV}$ channels of his OSO-3 instrument.

We believe that the extension of the solar X-ray spectrum above approximately $6 \mathrm{KeV}$ is strongly indicative of non-thermal processes taking place in the active regions on the Sun which were quiescent at the time of our flight. Indeed, the relatively flat spectrum extending to high energies beyond $12 \mathrm{KeV}$ may be bremsstrahlung from relativistic electrons either trapped or continually produced within the centres of activity. Further analysis of these data and additional measurements of the kind made during this flight show promise for helping to understand the physics of solar active regions.

Note added in proof: It has not yet been possible to reconcile the solar data of the three large-area systems for photon energies greater than $8 \mathrm{KeV}$. Therefore we consider it advisable to treat with caution the data above $8 \mathrm{KeV}$ from this experiment until such time as these particular measurements are better understood or until independent confirmation of a flat solar X-ray spectrum in the $8-22 \mathrm{KeV}$ interval is obtained.

\section{References}

Bowles, J.A., Culhane, J.L., Sanford, P.W., Shaw, M.L., Cooke, B.A. (1967) Planet. Space Sci., 15, 931.

Chodil, G., Jopson, R.C., Mark, Hans, Seward, F.D., Swift, C.D. (1965) Phys. Rev. Lett., 15, 605.

Chubb, T. A., Kreplin, R.W., Friedman, H. (1966) J. geophys. Res., 71, 3611.

Fritz, G., Kreplin, R.W., Meekins, J.F., Unzicker, A. E., Friedman, H.(1967) Astrophys. J., 148, L133.

Giacconi, R., Reidy, W.P., Zehnpfennig, T., Lindsay, J.C., Muney, W.S. (1965) Astrophys. J., 142, 1274.

Mathieson, E., Sanford, P.W. (1963) in Proc. Int'l. Symp. on Nuclear Elec., p. 65.

Peterson, L.E. (1967) Paper presented at the XIII General Assembly of the IAU, Prague, August 23, 1967. 
Peterson, L.E., Schwartz, D.A., Pelling, R.M., McKenzie, D. (1966) J. geophys. Res., 71, 5778. Pounds, K.A., Russell, P.C. (1966) in Space Research, VI, p. 38.

Reed, R.D., Bakke, J.C., Reagan, J.B., Acton, L.W. (1968) IEEE Trans. on Nuclear Sci., NS-16 (in press).

Underwood, J.H., Muney, W.S. (1967) Solar Phys., 1, 129.

Zhitnik, I. A., Krutov, V.V., Malyavkin, L.P., Mandel'shtam, S. L., Cheremukhin, G.S. (1967) Kosm. Issled., Akad. Nauk SSSR (Moscow), 5, 276. 